> Avec la navigation à vapeur, les hommes, mais aussi les germes pathogènes, se déplacent plus rapidement. En 1865, le pèlerinage à La Mecque est à l'origine d'une terrible pandémie de choléra. Celle-ci provoque un traumatisme profond et la communauté internationale va s'attacher à faire des pèlerins le principal « groupe à risque ». Elle leur impose un contrôle exemplaire, bien plus sévère que celui qui est appliqué à la navigation ordinaire et commerciale, entraînant l'édification d'un formidable dispositif quarantenaire dans la mer Rouge et au Levant, à l'heure où les quarantaines disparaissent partout ailleurs. En dépit de leur caractère coercitif, les pèlerins ne manifestent pas d'hostilité à l'égard des actes médicaux et prophylactiques en usage dans les lazarets, mais s'élèvent contre les modalités déshumanisées de leur réalisation. La pression conjuguée du nationalisme et des revendications saoudiennes ne mettra fin à ce dispositif qu'en 1957.<

\section{Les pèlerins \\ de La Mecque, \\ les germes \\ et la communauté \\ internationale}

\section{Sylvia Chiffoleau}

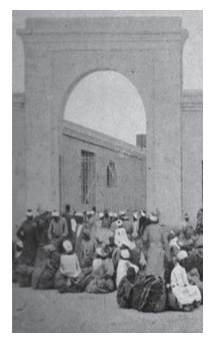

Institut français du Proche-Orient, BP 11-1424, Beyrouth, Liban. sylvia.chiffoleau@gmail.com

cède le pas aux navires à vapeur. Ceux-ci permettent de multiplier le nombre des pèlerins, notamment en provenance de l'Asie, jusqu'alors en nombre réduit en raison de la longueur et de la difficulté du voyage effectué par voilier dans des mers peu sûres. Mais en 1865, le choléra quitte l'itinéraire terrestre par l'Asie Centrale et la Russie qu'il avait emprunté jusqu'alors pour se répandre en Europe, puis dans le monde, à partir du port d'Alexandrie. Il suit les pas de pèlerins revenant du Hedjaz où ils avaient été contaminés par des coreligionnaires venus d'Inde, berceau de la maladie. La pandémie provoque un traumatisme profond. La communauté internationale, en cours de formation à cette époque, va dès lors s'attacher à faire des pèlerins le principal « groupe à risque » dans la propagation des maladies épidémiques. Elle leur impose un contrôle exemplaire, bien plus sévère que celui qui est appliqué à la navigation ordinaire et commerciale, entraînant l'édification d'un formidable dispositif quarantenaire dans la mer Rouge et au Levant, à l'heure où les quarantaines disparaissent partout ailleurs.

\section{La fabrication d'un groupe à risque}

Les premières conférences sanitaires internationales En 1851, à la faveur de l'apaisement relatif des tensions politiques européennes après 1815 [1], les puissances du Vieux Continent décident de se mobiliser pour lutter ensemble contre la menace des épidémies.

${ }^{1}$ Région ouest de l'actuelle Arabie saoudite, comprennant notamment les provinces de Tabuk, Médine, La Mecque et Al Bahah.
Les germes responsables des maladies infectieuses ont est resté dans toutes les mémoires. L'Europe du XIX ${ }^{e}$ sièportee par une idéologie de progrès, tend d'ab des fleurons de cette modernité triomphante, la navigation à vapeur, en augmentant la vitesse de déplacement le monde plus vulnérable encore au danger de propagation des maladies. La nouvelle technologie favorise certes le développement du commerce, mais aussi la mobilité des hommes. Le pèlerinage à La Mecque, qui de tout temps a constitué l'une des plus importantes migrations temporaires au monde, se trouve profondément bouleversé par l'apparition de ce nouveau moyen de transport. L'antique caravane chamelière, dont la durée du trajet constituait une prophylaxie naturelle, 
Elles donnent ainsi naissance à un processus qui aboutira, au siècle suivant, à la construction du système international qu'est l'OMS (organisation mondiale de la santé). Les deux premières conférences sanitaires internationales, en 1851 puis 1859 , ne réunissent en réalité que les pays européens et l'Empire ottoman, invité en tant que «zone tampon » entre les foyers asiatiques des maladies épidémiques, peste et choléra, et l'Europe. Elles visent d'une part à harmoniser les législations sanitaires des différents pays d'Europe, afin de les accorder aux exigences libérales d'un commerce maritime en pleine expansion, et d'autre part à mobiliser le dispositif sanitaire ottoman, nouvellement établi, pour le mettre au service de la lutte contre la menace épidémique [2]. En 1865, lorsque le choléra se répand dans le monde à partir du pèlerinage à La Mecque, l'événement donne un sens nouveau à ce second objectif. L'obsession sécuritaire des puissances, qui visait à bloquer les épidémies sur la rive orientale de la Méditerranée de façon à ce qu'elles ne puissent pénétrer en Europe, se referme sur le voyage sacré des musulmans et transforme en profondeur les objectifs des rencontres sanitaires internationales. Or, si l'épisode épidémique de 1865 a bien prouvé le potentiel de dangerosité de cette migration temporaire de masse, le succès de la promotion des pèlerins comme « groupe à risque » repose également sur une série de facteurs politiques.

\section{Le pélérinage de La Mecque:}

\section{entre enjeu sanitaire et politique coloniale}

Lorsque la communauté internationale en cours de formation s'empare de la question du pèlerinage à La Mecque, celui-ci n'est déjà plus une affaire strictement musulmane, mais relève désormais en partie de l'univers colonial. En Inde, en Algérie et dans l'Insulinde ${ }^{2}$, bien des musulmans deviennent des sujets coloniaux placés sous la tutelle de la Grande-Bretagne, de la France ou de la Hollande. L'emprise coloniale ne cesse de s'étendre ensuite jusqu'à inclure, après la Première Guerre mondiale, la quasi-totalité des peuples musulmans. Or le territoire sacré de l'islam, La Mecque, demeure inaccessible aux non-musulmans. Les puissances européennes craignent donc que le pèlerinage, carrefour de rencontre des croyants du monde entier, ne soit un lieu favorable à l'émergence et à la diffusion d'un discours politico-religieux qui viserait à remettre en cause leur pouvoir sur les pays colonisés. Les visées nationalistes des pèlerins sont en réalité plus fantasmées que réelles [3], mais les principales puissances coloniales européennes, qui sont aussi celles qui orchestrent le cycle des conférences sanitaires internationales, voient dans le contrôle sanitaire spécifique imposé aux pèlerins un gage supplémentaire de surveillance des flux. Ce contrôle vient en effet s'ajouter aux procédures administratives souvent sévères qu'édictent les puissances coloniales, chacune pour ses « sujets musulmans » désirant effectuer le voyage sacré.

Par ailleurs, les pèlerins n'apparaissent que comme un problème à gérer, et non comme une force humaine qui pourrait être mise au

${ }^{2}$ L'Insulinde ou Asie du Sud-Est insulaire, anciennement appelée Malaisie, archipel malais ou archipel indien. service des États et dont il conviendrait d'améliorer l'état général. C'est donc sans état d'âme et sans aucune visée altruiste que la jeune communauté internationale s'empare des pèlerins, soumis dès lors au même processus de dépersonnalisation dont sont victimes les « indigènes » en situation coloniale. Ils sont traités en masses anonymes, en listes et tableaux de chiffres : tant d'arrivées et de départs du lazaret, tant de morts par «nationalité ». En ce sens, le traitement qu'ils subissent apparaît plus insensible et plus dur que celui qui s'applique aux migrants, autre masse mobile jugée dangereuse. Les problèmes sanitaires liés aux migrants, en dépit de plusieurs tentatives de la part des États-Unis, ne sont réellement abordés dans le cadre des conférences sanitaires internationales qu'en 1926, et traités dès lors dans le cadre des conventions sanitaires. II est vrai que contrairement aux pélerins, les migrants, qui représentent une force de travail en marche, reçoivent une attention sanitaire de la part des pays de départ et d'arrivée, ainsi que des compagnies de navigation, qui limite considérablement les temps de quarantaine [4]. Dans les années 1930, en raison des filtres préalables, la station quarantenaire d'عllis Island dans la baie de New-York, ne retient plus que quelques milliers de migrants, alors que les quarantaines de la mer Rouge pour les pèlerins sont encore en pleine activité.

Enfin, les conférences sanitaires internationales sont loin d'être des réunions consensuelles, elles se font au contraire l'écho des multiples conflits scientifiques et politiques qui traversent le $x x^{e}$ et le début du $x x^{e}$ siècle. La question du caractère contagieux ou non des maladies épidémiques demeure ouverte jusqu'à la fin $\mathrm{du} X \mathrm{X}^{\mathrm{e}}$ siècle et soulève à chaque réunion de virulents débats. Les conférences sont également le reflet de la concurrence politique que se livrent entre elles les puissances européennes. La France et l'Angleterre, notamment, défendent deux visions différentes de la prophylaxie sanitaire : la première bataille pour le maintien d'un dispositif quarantenaire « raisonné », la seconde cherche à imposer le principe de la désinfection et du suivi individualisé des voyageurs. Dans ce contexte international naissant, qui révèle déjà de fortes divergences entre les acteurs, la question du pèlerinage à La Mecque apparaît finalement comme le plus petit dénominateur commun sur lequel peut se faire l'accord. La désignation de ce « groupe à risque » parvient même à convaincre les Anglais, opposés par principe à toute mesure de type quarantenaire, a fortiori si celle-ci doit s'appliquer sur les territoires qu'ils contrôlent. Or, c'est précisément le cas de l'isthme de Suez, considéré comme l'ultime barrage contre les épidémies avant que 
celles-ci ne viennent à menacer l'Europe. Pour les pèlerins, les Anglais sont prêts à faire une concession, dès lors qu'il s'agit d'un groupe qui ne revêt pas une importance majeure en termes d'intérêts commerciaux, et, qu'en contrepartie, leur navigation marchande bénéficie d'une plus grande libéralité lors de la traversée du canal de Suez. II est vrai également qu'il est assez aisé de faire admettre aux opinions publiques européennes un contrôle sur une foule anonyme jugée dangereuse, de surcroît attachée à une religion peu estimée, alors qu'elles sont très réticentes dès lors qu'il s'agit d'imposer des entraves sanitaires aux flux commerciaux et de voyageurs ordinaires.

\section{Deux poids, deux mesures dans le contrôle sanitaire des transports maritimes}

L'intérêt porté aux pèlerins ne relève donc pas de la seule logique scientifique, mais aussi de considérations politiques. Or l'accord établi sur la nécessité de focaliser la lutte contre les épidémies sur le groupe des pèlerins va être à l'origine de l'établissement d'un vaste dispositif quarantenaire en mer Rouge et au Levant et s'inscrire au centre de la législation sanitaire internationale. En1866, à la suite de la crise épidémique de l'année précédente, une troisième conférence sanitaire se réunit à Istanbul et adopte les principes du dispositif de protection à l'égard des flux du pèlerinage. II s'agit d'une part d'installer deux verrous quarantenaires, à l'entrée et à la sortie de la mer Rouge, et d'autre part d'encourager l'Empire ottoman à améliorer les conditions sanitaires et d'hygiène au Hedjaz même. En raison des tensions politiques intra-européennes, qui entravent le développement du processus international, il faut cependant attendre la septième conférence sanitaire, en 1892, pour voir adopter la première convention sanitaire internationale, quelque quarante ans après le début du cycle des conférences. À l'heure où la théorie des germes triomphe, la navigation commerciale obtient la liberté de circulation, notamment pour la traversée du canal de Suez qui se fait désormais sans quarantaine préalable. En revanche les navires qui transportent les pèlerins, toujours suspects, sont quant à eux systématiquement soumis à quarantaine dans les lazarets établis sur la mer Rouge, et ce avant même l'adoption de la législation sanitaire internationale. La pratique puis la loi établissent donc de facto un contrôle sanitaire à deux vitesses, faisant des frontières maritimes des «membranes poreuses » [5] qui laissent passer certains flux et non d'autres. Le dispositif restera en vigueur, avec certes des modifications, des améliorations et des allégements, jusque dans les années 1950. Le régime sanitaire spécial des pèlerins figure encore en annexe du premier règlement sanitaire international adopté par l'OMS en 1951.

\section{Un régime d'exception coercitif}

\section{Les lazarets de Camaran et Tor : établissements de quarantaine}

L'établissement du chemin de fer du Hedjaz, dans les premières années $\mathrm{du} x \mathrm{x}^{\mathrm{e}}$ siècle, puis l'apparition des routes terrestres dans les années 1930, susciteront à leur tour l'édification de lazarets et de stations sanitaires pour les pèlerins [6]. Cependant, en raison de l'importance des flux du pèlerinage par la voie maritime, c'est le dispositif maritime qui demeure le plus massif. Celui-ci est constitué principalement de deux immenses lazarets. L'un est établi en 1881 sur l'île de Camaran, dans le détroit de Bab al-Mandeb, et forme un filtre par lequel passent à l'aller tous les pèlerins arrivant des régions du Sud-Est asiatique (Inde, Insulinde, territoires malais et chinois) où se trouvent les foyers du choléra et de la peste, laquelle fait sa réapparition à l'extrême fin du XIX siècle. L'autre, destiné aux pèlerins du nord (Maghreb, Asie Centrale et Russie, Turquie et Syrie) sur le retour, est fondé au cours des années 1870 à Tor, localité située à l'extrémité sud de la péninsule du Sinaï. Ces lazarets sont de gigantesques villes éphémères, réactivées chaque année pendant les deux ou trois mois de ce que l'on nomme «la campagne quarantenaire ».

Lors du pèlerinage musulman annuel, deux cas de figure peuvent se présenter : soit le pèlerinage est net, c'està-dire qu'aucune maladie épidémique ne s'y est manifestée, soit il est brut quand des cas de choléra ou de peste ont été déclarés lors des cérémonies religieuses à La Mecque. Dans le premier cas, la halte aux lazarets de Camaran et Tor est de courte durée, trois jours environ, dans le second cas, la quarantaine peut s'éterniser plusieurs semaines. Ce n'est qu'à partir des années 1920, et surtout 1930, avec le recul du péril épidémique, que les temps d'observation seront sensiblement diminués. Mais de 1891 à 1918, date à laquelle le choléra disparaît du Hedjaz, on ne compte que six années nettes sur vingt-huit ; la plupart des pèlerins qui font le voyage sacré au cours de cette période sont donc amenés à connaître le lazaret, soit plusieurs dizaines de milliers de personnes chaque année. En outre, au tournant du siècle, la conjonction de la peste et du choléra, en Inde comme au Hedjaz, rend les mesures plus drastiques encore.

Le temps de la quarantaine : un affront à l'individu Conçus avant la révolution bactériologique, les lazarets de la mer Rouge n'ont d'abord eu d'autre finalité que l'enfermement; il s'agissait de laisser s'éteindre les épidémies dans ces régions désertiques. Très vite cependant, des mesures de désinfection sont apparues, puis sont devenues de plus en plus exigeantes et sophistiquées à mesure de l'ancrage de la théorie des germes (Figure 1). Si, au début, les effets des pèlerins ne semblent avoir été qu'irrégulièrement désinfectés par aspersion de produits désinfectants, l'installation d'une première étuve à Tor (Figure 2) en 1889, instaure un rituel très codifié de la désinfection. À leur arrivée, les pèlerins sont dirigés vers le bâtiment de désinfection, leurs vêtements et leurs bagages leur sont retirés, triés et vérifiés, puis placés dans d'immenses 


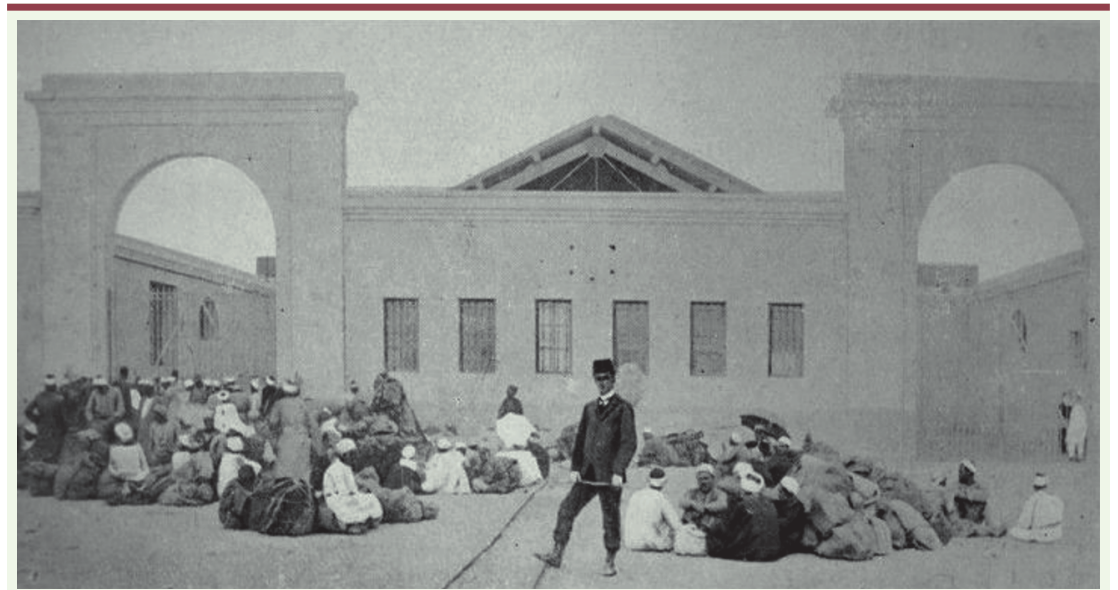

Figure 1. Entrée du bâtiment de désinfection, Tor. Tiré de Briend René, Établissements quarantenaires, Alexandrie, Société de publications égyptiennes, s.d.

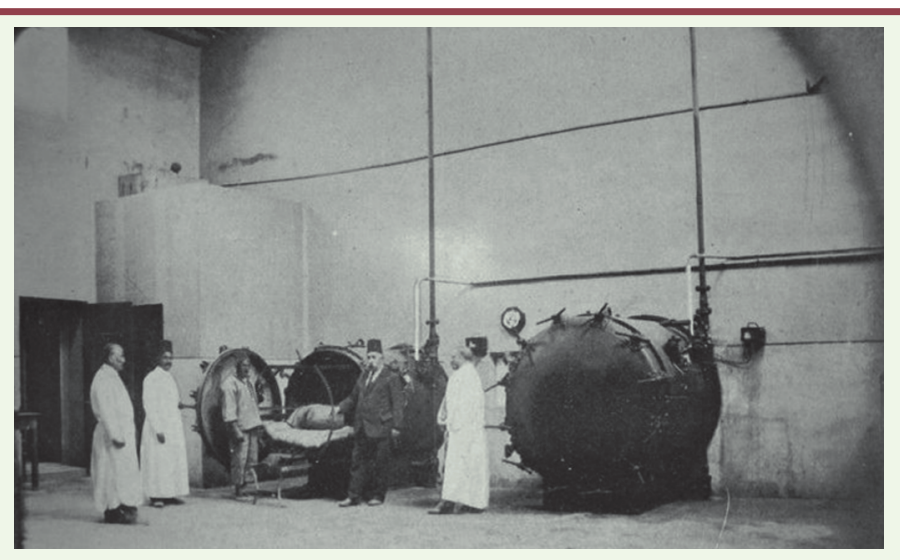

Figure 2. Salle des étuves, station des Sources de Moïse. Tiré de René Briend, Établissements quarantenaires, Alexandrie, Société de publications égyptiennes, s.d.

étuves à vapeur pendant qu'eux-mêmes sont invités à procéder à un bain de mer. Au tournant du siècle, des douches à eau de mer sont introduites dans les lazarets (Figure 3), ainsi que des bains pour les voyageurs de première classe. En cas de pèlerinage brut, toutes les denrées transportées par les pèlerins sont détruites dans un vaste incinérateur.

Accueillis comme «suspects » à leur arrivée, les pèlerins peuvent, au terme de cette séquence de désinfection, se rendre dans la partie du lazaret dite «propre » où se trouvent les hébergements et les structures hospitalières. Les sections où les pèlerins purgent leur quarantaine sont organisées par bateau et étroitement surveillées par des soldats pour empêcher toute communication avec l'extérieur ou entre les sections. À Tor, elles sont constituées de tentes de l'armée égyptienne, à Camaran de huttes de roseau, et dans les deux cas dépourvues de tout ameublement ; ceux qui n'ont pas apporté de tapis doivent se contenter du sol nu. Mais le confort importe peu aux promoteurs de ce système qui ne veulent y voir qu'un dispositif sécuritaire régi par la salubrité et qui redessine sans état d'âme les frontières entre le pur et l'impur.
Pour les pèlerins, le temps de la quarantaine s'écoule en discussions et pieuses lectures, interrompues par les nécessités de l'observation médicale à laquelle ils sont soumis. Plus on s'avance dans le vingtième siècle, plus les lazarets deviennent aussi des laboratoires pour la médecine de masse, révélateurs des impasses de celle-ci. En principe, les médecins du lazaret assurent une visite médicale quotidienne auprès de chaque pèlerin. En réalité, celle-ci se réduit bien souvent à un simple coup d'œil jeté à un visage, accompagné d'une furtive prise de pouls. Les personnes malades sont envoyées dans les hôpitaux du lazaret où elles sont prises en charge, mais elles se heurtent à une communication difficile avec le corps médical, liée bien souvent à une mutuelle incompréhension linguistique. À partir du début $d u x x^{e}$ siècle, les vaccinations anticholériques et antipesteuses sont largement pratiquées, en dépit des doutes sur leur efficacité. Plus tard, les examens bactériologiques des selles le sont également, bien que la législation internationale ne les rende pas obligatoires. Au terme de leur quarantaine, les pèlerins rejoignent leur bateau, lequel a subi également une désinfection totale, laissant derrière eux de nombreux morts, plus souvent victimes de maladies ordinaires ou d'épuisement que des épidémies dont la crainte a suscité l'implantation d'un tel système.

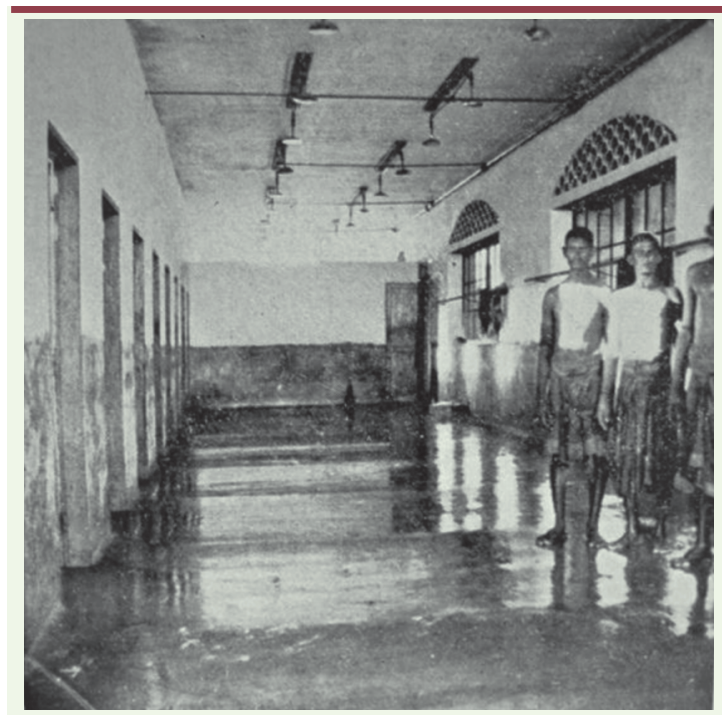

Figure 3. Douches à Camaran. Tiré du rapport de la commission d'inspection des lazarets sur le lazaret de Camaran, Dr Clemow rapporteur, Constantinople, 1906. 
En dépit de leur prégnance et de leur caractère coercitif, les pèlerins ne manifestent pas d'hostilité directe à l'égard des actes médicaux et prophylactiques. C'est le principe même de l'enfermement, ainsi que la façon autoritaire et peu respectueuse de l'individu avec laquelle sont réalisées les procédures sanitaires dans un contexte de masse, qui soulèvent l'indignation. Le mépris, le manque de considération à l'égard des pèlerins, la négligence avec lesquels on traite leurs affaires (les bagages, notamment, sont souvent considérablement endommagés par l'opération de désinfection) provoquent maintes protestations. Et celles-ci sont plus vives encore quand il s'agit des aspects directement matériels de la vie au lazaret; quand celui-ci manque de pain ou que les provisions sont trop coûteuses, on frôle l'émeute. Avec la montée en puissance du nationalisme et la prise de conscience concomitante des risques politiques attachés à une trop grande désinvolture à l'égard des « indigènes », du côté des puissances européennes, le terme «humanité » finit par pénétrer dans le vocabulaire international sur le dossier des lazarets. D'ailleurs, les efforts d'équipement de ceux-ci visent désormais explicitement le confort des pèlerins.

Mais l'existence et les pratiques des lazarets ne sont pas seulement un affront aux individus, elles sont aussi une atteinte à la souveraineté saoudienne. Depuis la naissance du royaume, les autorités saoudiennes n'ont eu de cesse de prouver leur capacité à assurer la sécurité sanitaire du pèlerinage afin de lever ce système d'exception. Ce n'est cependant qu'en 1957 que la méfiance internationale cède enfin et que les mesures de contrôle dévolues aux stations de Tor et Camaran sont intégralement et définitivement transférées aux services quarantenaires de Djeddah, mettant un terme à un siècle de contrôle sanitaire partagé du pèlerinage à La Mecque. $\diamond$

\section{SUMMARY}

Pilgrims to Mecca, germs

and the international community

With steam navigation, men, but also seeds of diseases, move faster. In 1865 , the pilgrimage to Mecca induced a terrible cholera pandemic. This caused deep trauma in Europe and prompted the international community, recently born, to focus on the pilgrims who were regarded as the main "risk group" in the spread of epidemics. Through a series of international sanitary conferences, European powers, in agreement with the Ottoman Empire, imposed a heavy control over the pilgrims, much harsher than that applied to ordinary commercial navigation exchanges. These decisions led to the establishment of a huge quarantine device in the Red Sea and the Levant, including two lazaretto, one at the entrance and the other at the exit of the Red Sea, where the pilgrims were trapped and disinfected during their sacred journey. This contrasted with the concommitant disappearance of quarantines everywhere else. The pilgrims did not show hostility towards medical and prophylactic treatments but protested against the dehumanizing conditions of their realization. The combined pressures of nationalism and sovereignty claims from Saudi Arabia ended this system in 1957. $\diamond$

\section{CONFLIT D'INTÉRÊTS}

L'auteur déclare n'avoir aucun conflit d'intérêts concernant les données publiées dans cet article.

\section{RÉFÉRENCES}

1. Harrison M. Disease, diplomacy and international commerce: the origins of international sanitary regulation in the nineteenth century. J Global History $2006 ; 1$ : 197-217.

2. Howard-Jones N. Les bases scientifiques des conférences sanitaires internationales (1851-1938). Genève : OMS, 1975.

3. Chiffoleau $S$. Le pèlerinage à La Mecque à l'époque coloniale : matrice d'une opinion publique musulmane? In: Chiffoleau S, Madoeuf A, eds. Les pèlerinages au Maghreb et au Moyen-Orient. Espaces publics, espaces du public. Beyrouth : IFPO, 2005 : 131-63.

4. Markel H. The eyes have it: trachoma, the perception of disease, the United States Public Health Service, and the American Jewish immigration experience, 1897-1924. Bull Hist Med $2000 ; 74:$ 525-60.

5. Huber $V$. The unification of the globe by disease? The international sanitary conferences on cholera, 1851-1894. Hist J $2006 ; 49: 453-76$.

6. Delamare G. La défense sanitaire de la ligne Médine-Damas. Constantinople : Imprimerie L. Mourkidès, 1912.

\section{TIRÉS À PART}

S. Chiffoleau

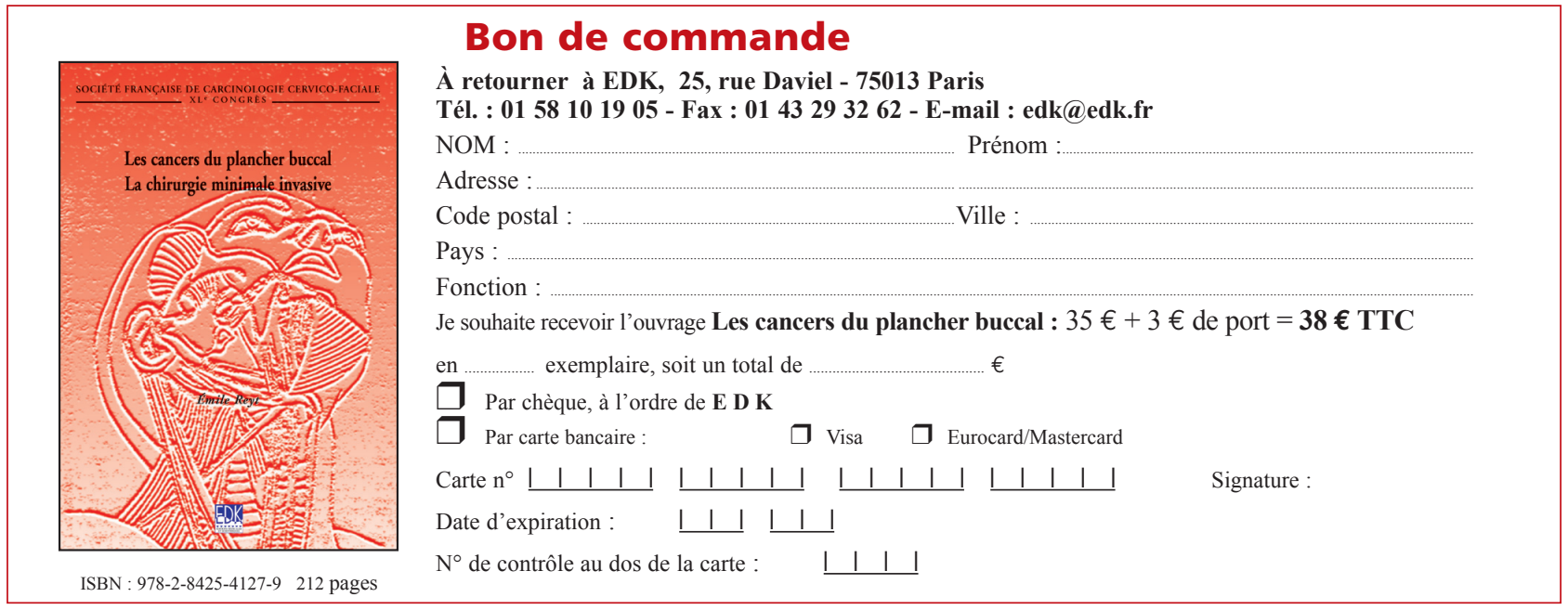

